\title{
Examining the role of insulin in the regulation of cardiovascular health
}

\section{SUMMARY}

A substantial body of evidence has reported insulin has direct actions on the cardiovascular system independent of its systemic effects on plasma glucose or lipids. In particular, insulin regulates endothelial synthesis of the vasoactive mediators nitric oxide and endothelin-1, yet the importance of this in the maintenance of cardiovascular health remains poorly understood. Recent studies using animals with targeted downregulation of insulin signaling in vascular tissues are improving our understanding of the role of insulin in vascular health. This article focuses on the direct actions of insulin in cardiovascular tissues, with particular emphasis on the molecular mechanisms of insulin action on endothelial function. The potential contribution of impaired vascular insulin action to the cardiovascular complications of diabetes will also be discussed.

\section{KEYWORDS}

Insulin, diabetes, endothelium, nitric oxide, endothelin, cardiovascular 
Insulin is a key hormonal regulator of glucose homeostasis, acting to store excess plasma glucose as glycogen in muscle and liver or triglyceride in adipose tissue. To achieve this, insulin stimulates glucose uptake in striated muscle and adipocytes, glycogenesis in liver and muscle, glucose phosphorylation in liver and suppresses hepatic gluconeogenesis. Furthermore, insulin has marked effects on lipid metabolism, including the stimulation of fatty acid and triglyceride synthesis and suppression of lipolysis [1].

Diabetes mellitus is characterized by insulin insufficiency due to autoimmune destruction of insulin-secreting $\beta$-cells or systemic insulin resistance. Obesity is a central factor in the development of insulin resistance, when the capacity of adipocytes to store triglyceride is exceeded, leading to inappropriate lipid storage in other tissues, including skeletal muscle and the liver $[1,2]$. Metabolites of this ectopic lipid and increased circulating fatty acid concentrations impair insulin sensitivity in target tissues. In parallel, infiltration of adipose tissue with macrophages and other leukocytes occurs, promoting a chronic, subclinical pro-inflammatory environment in which pro-inflammatory cytokines also impair insulin sensitivity [2]. To maintain glycemia, insulin resistance leads to compensatory hyperinsulinemia prior to the development of frank diabetes. As a consequence, patients with diabetes exhibit hyperglycemia and dysregulated lipid metabolism and storage.

Insulin resistance and diabetes are associated with a markedly increased risk of developing macrovascular and microvascular cardiovascular disease. Indeed, cardiovascular diseases are the principal cause of morbidity and mortality in patients with diabetes [101]. The mechanisms that underlie the predisposition of people with diabetes to cardiovascular disease are, however, incompletely understood although multiple lines of evidence indicate that endothelial dysfunction, characterized by reduced nitric oxide (NO) bioavailability, is a key early step in the development of the cardiovascular complications of diabetes [3]. The abnormal glucose and lipid metabolism observed in patients with insulin resistance/diabetes in addition to increased circulating proinflammatory cytokines promote cardiovascular disease, yet substantial 
evidence demonstrates that insulin directly influences cardiovascular biology independent of its metabolic actions. Furthermore, the direct cardiovascular actions of insulin may be impaired in insulin resistance and diabetes, such that vascular insulin resistance co-exists with systemic insulin resistance.

This article describes the direct effects of insulin on the cardiovascular system, with a particular emphasis on the current understanding of insulin signaling and actions in blood vessels in normal physiology and insulin resistance.

\section{Insulin signaling}

Insulin binding to the dimeric insulin receptor (IR) tyrosine kinase stimulates autophosphorylation of the intracellular domains and the resultant phosphotyrosine motifs serve as docking sites for adapter proteins including the insulin receptor substrate (IRS) proteins, Shc (Src-homology and collagen-like) proteins and APS (adaptor protein with pleckstrin homology and Src homology 2 domains) $[4,5,6]$. This simple ligand-receptor binding model is complicated by the closely-related insulin-like growth factor-1 (IGF-1) receptors (IGFRs), which can form heterodimers with the IR, leading to the possible expression of IR homodimers, IGFR homodimers and IR-IGFR heterodimers on the cell surface, all of which may respond to insulin or IGF-1 with different specificity [6].

Upon binding to phosphotyrosine residues on the activated IR, IRSS are phosphorylated by the IR on tyrosine residues, generating docking sites for the recruitment of the lipid kinase phosphatidylinositol 3'-kinase (PI3K), which generates phosphatidylinositol-3,4,5-trisphosphate $\left(\mathrm{PIP}_{3}\right)$. The newly-synthesized $\mathrm{PIP}_{3}$ recruits the serine/threonine protein kinase Akt (also known as protein kinase B) to the plasma membrane, and Akt is subsequently phosphorylated and activated by phosphoinositide-dependent kinase 1 (PDK1) and mammalian target of rapamycin complex 2 (mTORC2) (Figure 1). The IR-IRS-PI3K-Akt pathway is the principal signaling 
pathway by which insulin exerts the majority of its metabolic effects including stimulation of glucose transport and inhibition of gluconeogenesis [4,5].

Insulin stimulation of IR stimulates binding of growth factor receptor-bound protein 2 (Grb2) to Shc, which subsequently activates the guanine nucleotide exchange factor Son of Sevenless (SOS). Activated SOS stimulates the small G-protein Ras, which initiates the RafMEK-ERK1/2 protein kinase cascade (Figure 1), associated with the mitogenic, growthpromoting actions of insulin $[4,7]$.

APS recruitment to phosphotyrosine motifs on the activated insulin receptor initiates recruitment of a complex containing $\mathrm{c}-\mathrm{Cbl}$ and $\mathrm{c}-\mathrm{Cbl}$-associated protein (CAP). Tyrosine phosphorylation of c-Cbl by the insulin receptor causes the recruitment of an adaptor protein (CRK) and the guanine nucleotide exchange factor C3G to the plasma membrane. C3G subsequently activates the small GTPase TC10, involved in the modulation of insulin-regulated membrane trafficking [5].

\section{Vascular effects of insulin}

The best characterized action of insulin in blood vessels is to stimulate vasodilation. Key early studies demonstrated increased leg [8] or forearm [9] blood flow in response to high physiological concentrations of insulin in healthy volunteers under euglycemic conditions, showing an independence from effects on glucose metabolism. Furthermore, the increase in blood flow was attenuated by an inhibitor of endothelial NO synthase (eNOS), suggesting insulin-stimulated vasodilation was NO-dependent $[8,9]$. In healthy individuals, acute infusions with insulin has little effect on systemic blood pressure, suggesting that increased insulin may increase blood flow to limb skeletal muscle in a tissue-specific manner. Increased limb blood flow is likely to represent the coordinated action of insulin to rapidly stimulate capillary recruitment in muscle via dilation of small arterioles and a slower vasodilation of larger vessels $[7,10]$. Indeed, increased capillary recruitment is sensitive to eNOS inhibition and was observed 
to occur within 5 minutes in rats infused with insulin, before any changes in blood flow [10]. The increased muscle blood flow in response to insulin subsequently leads to increased supply of nutrients to muscle, permitting further insulin-stimulated glucose uptake and glycogenesis $[7,10]$. Insulin has been demonstrated to have specific effects on endothelial cells, vascular smooth muscle and leukocytes associated with blood vessels and the cell-specific effects of insulin with respect to blood vessels are examined in greater depth later in this review.

\section{Cardiac actions of insulin}

The best-characterized role of insulin in the heart is to regulate substrate utilization. As in skeletal muscle, insulin stimulates GLUT4-mediated glucose uptake in the heart via the PI3KAkt and CAP-Cbl pathways and also stimulates glucose oxidation $[5,11]$. Insulin also reduces fatty acid oxidation and utilization, despite increasing fatty acid uptake, allowing a switch from fatty acid to glucose oxidation upon insulin stimulation. This metabolic flexibility for ATP production in the heart is important to permit it to respond to the fluctuations in energy substrate supply that occur during intense exercise and the switch between fed and fasted states $[1,11]$. The key metabolic role of insulin in the heart has been supported by studies demonstrating impaired mitochondrial function and decreased flux through the TCA cycle with increased oxidative stress in hearts of mice with a cardiomyocyte-specific deletion of the IR [12].

Insulin has been reported to increase myocardial microvascular blood flow in healthy men [13], suggesting a similar effect to that seen in peripheral blood vessels and also has a positive inotropic effects in the human atrial myocardium that are sensitive to PI3K inhibitors [14]. Furthermore, insulin stimulation of the PI3K-Akt pathway has been reported to regulate numerous other cardiac processes, including hypertrophy, apoptosis and autophagy, as reviewed elsewhere $[11,15]$. Insulin infusion is used in the clinical setting to improve outcomes in acute coronary patients, yet the mode of action and efficacy of this is still uncertain [15].

\section{Effects of insulin on platelets and erythrocytes}


Release of ATP from erythrocytes has been proposed to regulate blood flow distribution, particularly that of the cerebral and skeletal muscle microcirculation, due to activation of vascular endothelial cell purinergic receptors which subsequently stimulate NO production [16]. Incubation of erythrocytes from healthy volunteers with insulin ex vivo has been demonstrated to have no effect on basal ATP synthesis but rapidly attenuated ATP release stimulated by reduced oxygen tension, impairing their ability to dilate rodent arterioles [17]. This study therefore indicates that insulin may have a detrimental effect on erythrocyte-mediated regulation of blood flow in the microcirculation, yet the effect of insulin on erythrocyte function in vivo is poorly characterized and requires more research.

In contrast, insulin has been reported to stimulate ATP and adenosine release from platelets from healthy individuals ex vivo in a manner sensitive to inhibitors of NO synthesis [18]. Furthermore, stimulation of NO synthesis by insulin inhibits platelet aggregation and insulin infusion improved platelet responses to NO donors in volunteers with diabetes and acute coronary syndromes [19]. Platelet-derived adenosine/ATP might therefore contribute to insulinstimulated vasodilation and blood flow, whereas insulin-stimulated NO release may also reduce platelet aggregation.

\section{The effect of insulin on the endothelium}

As described above, insulin stimulates vasodilatation in a manner sensitive to inhibitors of NO synthesis. In addition to this well-described stimulation of NO synthesis by insulin, insulin also stimulates synthesis of the vasoconstrictor, endothelin-1 (ET-1), which can dominate under certain circumstances [20]. The molecular mechanisms by which insulin stimulates NO and ET1 synthesis are discussed further below.

\section{Insulin stimulation of endothelial NO synthesis}

$\mathrm{NO}$ is synthesized from L-arginine, NADPH and $\mathrm{O}_{2}$, by eNOS, with L-citrulline and $\mathrm{NADP}^{+}$formed as co-products. The activity of eNOS is regulated by multiple mechanisms, 
including allosteric activation, post-translational modification and subcellular localisation [21]. Insulin-stimulates NO synthesis in cultured human endothelial cells and much research has characterized the mechanism by which this occurs. Insulin-stimulated NO synthesis in cultured endothelial cells is independent of $\mathrm{Ca}^{2+}$ concentration but dependent on IRS1-PI3K-PDK1-Akt pathway activation $[1,3,7]$. IGF-1 has been reported to be a markedly less effective stimulus for NO synthesis - indeed, recent elegant studies using mice with an endothelial-specific knockdown of IGFR or overexpression of IGFR have reported that the IGFR is a negative regulator of insulin-stimulated NO synthesis, perhaps due to formation of IR-IGFR heterodimers that are less sensitive to insulin than IR homodimers [22,23]. eNOS is subject to reversible phosphorylation at several sites [24], and Akt-mediated phosphorylation of eNOS at Ser1177, which reduces the requirement for $\mathrm{Ca}^{2+}$ and permits $\mathrm{NO}$ synthesis in the absence of increased cellular $\mathrm{Ca}^{2+}$ is required for insulin-stimulated NO synthesis in cultured endothelial cells (Figure 2) $[7,24]$. Furthermore, Akt-mediated phosphorylation of eNOS Ser615 synergizes with Ser1177 phosphorylation, contributing to insulin-stimulated NO synthesis [25]. However, insulinstimulated NO synthesis has been reported to be inhibited in the absence of altered insulinstimulated eNOS phosphorylation, indicating that insulin stimulates NO synthesis via both eNOS phosphorylation-dependent and phosphorylation-independent mechanisms [25,26].

Dimerisation of eNOS is required for activity and other proteins have been reported to interact with and regulate eNOS, including caveolin-1 and Hsp90 [27]. The association with caveolin-1 inhibits eNOS activity [28], whereas association with Hsp90 has been reported to promote eNOS activity [27]. Insulin has been reported to promote Hsp90-eNOS association in bovine endothelial cells, and the disruption of Hsp90-eNOS binding inhibited insulin-stimulated eNOS activation $[7,29]$. Furthermore, stimulation of human endothelial cells with insulin has been reported to increase transport of the eNOS substrate L-arginine in an Akt-dependent manner [26]. In agreement with a role for L-arginine transport in insulin-stimulated NO synthesis, inhibition of transport with lysine has been reported to inhibit insulin-stimulated 
umbilical vein relaxation [30]. Asymmetric dimethylarginine (ADMA) is a naturally occurring, competitive inhibitor of eNOS and elevated ADMA concentrations have been reported in subjects with diabetes [31]. Whether insulin regulates ADMA concentrations or ADMA influences insulin sensitivity in the endothelium has not been characterized.

Reactive oxygen species (ROS), such as superoxide react with NO to form peroxynitrites, thereby directly reducing NO bioavailability (Figure 2)[32]. Furthermore, superoxide-mediated oxidation of the eNOS cofactor tetrahydrobiopterin $\left(\mathrm{BH}_{4}\right)$ reduces the activity of eNOS, which, in turn, leads to uncoupling of eNOS, resulting in further superoxide synthesis [32]. Insulin has been reported to inhibit myocardial peroxynitrite formation after ischemia/reperfusion [33] and transgenic mice with endothelium-targeted overexpression of a dominant-negative mutant IR exhibited increased endothelial superoxide generation [34], yet little is known concerning the action of insulin on ROS synthesis in endothelial cells under basal conditions. Insulin has been reported to increase cellular concentrations of $\mathrm{BH}_{4}$ and $\mathrm{mRNA}$ expression of guanosine triphosphate cyclohydrolase-1, the rate-limiting enzyme for the synthesis of $\mathrm{BH}_{4}$, in cultured mouse brain microvascular endothelial cells [35]. Any or all of these phosphorylation-independent mechanisms of insulin may therefore contribute to insulinstimulated NO production.

Insulin has also been demonstrated to increase the expression of eNOS at the mRNA and protein level in cultured endothelial cells [7, 36], supported by the reduced eNOS expression observed in endothelial cells from mice with an endothelial-specific deletion of the IR [37] Furthermore, insulin has been demonstrated recently to increase the expression of argininosuccinate synthase, a key enzyme in the recycling of eNOS-derived citrulline to arginine [36]. These data suggest that insulin may not only increase eNOS expression but co-ordinate this with increased substrate supply by increasing recycling of citrulline to arginine. 
Some reports have reported that insulin may also engage mechanisms to terminate or limit eNOS activation. Post-translational modification of eNOS by myristoylation and palmitoylation permits dynamic association with specific subcellular compartments including the Golgi complex and caveolae $[27,28]$. Insulin rapidly increases eNOS palmitoylation, which is required for the traffic of eNOS and caveolin-1 from a perinuclear Golgi compartment to the plasma membrane [28]. Inhibition of eNOS palmitoylation also increases insulin-stimulated NO synthesis, indicating that insulin-stimulated trafficking of eNOS to caveolae may act to terminate insulin-stimulated NO synthesis [28]. Insulin has also been reported to attenuate the activity of eNOS via proline-rich tyrosine kinase 2-stimulated phosphorylation of eNOS at Tyr657 in mouse lung endothelial cells, which the authors argue may be a mechanism to limit excessive NO production [38].

\section{Insulin regulation of ET-1 synthesis}

Active ET-1 is synthesized by ET converting enzymes and stored in Weibel-Palade bodies within endothelial cells. Upon stimulation, these storage granules traffic to the plasma membrane to release ET-1 by exocytosis. ET-1 subsequently has autocrine or paracrine effects mediated by ET receptors on endothelial cells and vascular smooth muscle cells (VSMCs) [39]. Insulin-stimulated secretion of ET-1 is PI3K-independent but ERK1/2-dependent in endothelial cells, yet the precise signaling mechanisms that underlie insulin-stimulated ET-1 secretion remain uncertain (Figure 2)[3,7,39,40]. Control of vascular tone by insulin therefore appears to be regulated by the balance of NO and ET-1 secretion in response to insulin, as vasodilator actions of insulin are enhanced by ET-1 receptor blockade in humans and animals $[3,7]$. Furthermore, it is clear that NO regulates ET-1 synthesis and vice versa $[7,41]$.

\section{Other endothelial effects of insulin}

In addition to relaxation of underlying smooth muscle, endothelial PI3K activation and NO synthesis promotes endothelial cell proliferation and migration and suppresses leukocyte adhesion to endothelial cells (Figure 2) $[7,21,42]$. This latter effect is mediated, at least in part, 
by reduced chemokine and adhesion molecule expression due to attenuated proinflammatory signaling via the transcription factor nuclear factor $\kappa B(\mathrm{NF} \kappa \mathrm{B})$ [43], although the molecular mechanism(s) by which endothelial NO suppresses $\mathrm{NF} \kappa \mathrm{B}$ remain poorly characterized. Cultured human endothelial cells stimulated with insulin in the absence of proinflammatory stimuli exhibited inhibited expression of intercellular adhesion molecule-1 (ICAM-1) in a NO-dependent manner, inhibited $\mathrm{NF} \kappa \mathrm{B}$ activity and reduced expression of the proinflammatory chemokine, monocyte chemoattractant protein-1 (MCP-1) $[7,44]$. The anti-atherogenic actions of insulin will be discussed in greater detail later in this review.

Finally, it has been suggested that insulin action is limited by the rate of insulin entry into skeletal muscle. Insulin has been reported to stimulate its own transport across the endothelium to underlying skeletal muscle by an IR-mediated pathway [45]. As a consequence, stimulation of transendothelial insulin transport may therefore contribute to insulin-stimulated glucose uptake in muscle $[45,46]$.

\section{Effects of insulin in vascular smooth muscle}

In the intact vessel, insulin-stimulated synthesis of NO or ET-1 from endothelial cells elicits relaxation or constriction of underlying smooth muscle (Figure 2). NO binds to soluble guanylate cyclase in VSMCs, leading to increased CGMP production and activation of CGMPdependent protein kinase (PKG), ultimately stimulating vasorelaxation [41]. On the other hand, ET-1 binds to ET receptors on the VSMC surface, engaging multiple signaling pathways that stimulate contraction [41]. ET-1 also stimulates VSMC proliferation and migration, whereas NO attenuates VSMC proliferation [41]. Insulin may therefore regulate VSMC proliferation via the endothelium by the balance of insulin-stimulated NO and ET-1 synthesis (Figure 2).

In addition to these effects mediated by insulin's endothelial actions, insulin has direct effects on VSMCs, stimulating relaxation, attenuating contractility and reducing $\mathrm{Ca}^{2+}$ influx [47]. VSMCs have been reported to express the IR, eNOS and inducible NOS (iNOS). Indeed, insulin 
stimulation of isolated VSMCs increases NO synthesis by eNOS and iNOS in a PI3K-dependent manner, leading to relaxation via activation of PKG $[47,48]$. Insulin inhibits activation of the small GTPase, RhoA in a NO/cGMP-dependent manner, leading to increased myosin light chain phosphatase (MLCP) activity $[47,49]$. These effects, along with inhibition of $\mathrm{Ca}^{2+}$ influx and increased $\mathrm{Ca}^{2+}$ efflux are likely to underlie the direct effects of insulin on VSMC relaxation and contractility [47]. Finally, insulin has been reported to stimulate VSMC proliferation in an ERK1/2-dependent manner [50], yet VSMCs from mice lacking the IR demonstrated increased proliferation and migration rates, although this was associated with increased ERK1/2 signaling [51]. In summary, it can be seen that insulin may influence VSMC tone and proliferation by both direct and endothelium (NO and ET-1)-mediated effects.

\section{Regulation of vascular leukocytes by insulin}

Despite the importance of macrophages resident in the blood vessel wall and circulating monocytes in the development of vascular disease, very little is known of the role of insulin in vascular leukocytes. Insulin stimulates the IR/IRS2/PI3K/Akt signaling cascade in macrophages [52], and insulin has recently been reported to inhibit the mRNA expression of the proinflammatory chemokine MCP-1 in mouse peritoneal macrophages, an effect that was ablated in macrophages lacking IRS2 [53]. The same study reported increased macrophage accumulation in the vascular wall of mice transplanted with $\mathrm{IRS}^{-/}$bone marrow, indicating physiological concentrations of insulin are sufficient to suppress proatherogenic macrophage accumulation in the absence of proinflammatory stimuli [53]. The functional consequences of insulin stimulation in vascular leukocytes in the healthy blood vessel are poorly characterized and require further research.

\section{Insulin and perivascular adipose}


It has recently become apparent that the thin layer of adventitia-associated perivascular adipose tissue (PVAT) found in small arteries and arterioles signals to and influences blood vessel biology [54]. PVAT responds to insulin in much the same way as other adipose tissue and enlargement of PVAT is observed in obesity and type 2 diabetes [54,55]. PVAT synthesizes a number of cytokines and chemokines, including proinflammatory tumor necrosis factor- $\alpha$ (TNF $\alpha$ ), interleukin-6 (IL6) and MCP-1 as well as anti-inflammatory adiponectin. As PVAT expands, increased free fatty acid synthesis, due to reduced anti-lipolytic effects of insulin and increased infiltration of PVAT with macrophages leads to increased synthesis of proinflammatory cytokines and reduced synthesis of adiponectin [54]. PVAT may therefore form an important site of regulation of vascular function that is altered in obesity or type 2 diabetes.

\section{Attenuation of atherogenesis by insulin}

Atherosclerosis is regarded to be a chronic inflammatory disease, the early stages of which involve accumulation of low-density lipoprotein $(L D L)$ in the subendothelial space, increased ROS in the vessel wall and increased chemokine synthesis and adhesion molecule expression in endothelial cells. Subsequently, leukocytes adhere to and migrate across the endothelium, forming foam cells which further increase proinflammatory signaling, migration and proliferation of VSMCS and synthesis of connective tissue leading to the formation of a mature atherosclerotic plaque. Ultimately plaque rupture or erosion results in thrombosis and the occlusion of arteries $[4,7]$.

NO has anti-atherogenic actions, attenuating monocyte adhesion and VSMC proliferation, key early events in the development of atherosclerotic plaques. Therefore, if insulin does indeed stimulate biologically-relevant concentrations of NO, insulin should act to attenuate atherosclerosis. In support of this, insulin, in the absence of proinflammatory stimuli, inhibited expression of ICAM-1 in a NO-dependent manner, inhibited activity of the key proinflammatory transcription factor NF $\kappa B$ and reduced expression of MCP-1 in human endothelial cells $[7,44]$. 
Administration of insulin for 3 days has been reported to decrease intimal area and increased re-endothelialization after balloon catheter injury in rat arteries, without altering plasma glucose concentrations significantly [56]. These studies support an anti-atherogenic action of insulin, yet care should be taken when assessing studies with systemic, prolonged treatment with insulin which may mimic hyperinsulinemia, with potential detrimental effects on vascular health.

Recent studies using mice in which vascular insulin signaling have been ablated or downregulated have been particularly enlightening with respect to the effect of insulin on atherogenesis. Atherosclerosis-prone mice transplanted with bone marrow from animals lacking the IR developed larger more complex atherosclerotic lesions with greater necrotic cores than animals transplanted with wild type bone marrow [57]. Furthermore, the macrophages lacking the IR showed increased apoptosis upon cholesterol loading and increased expression of scavenger receptor A [57], indicating loss of insulin signaling increases uptake of modified atherogenic LDL by macrophages and increases the severity of lesions which are more likely to cause acute coronary artery disease. In contrast to the study of Han and colleagues, deletion of insulin receptors in myeloid lineage cells (macrophages and neutrophils) decreased the size of lesions in atherosclerosis-prone mice [58]. The reasons for the observed differences may be the different cell types affected or the different diets and genetic backgrounds of the mice used.

In atherosclerosis-prone mice with targeted deletion of insulin receptors in the vascular endothelium, atherosclerotic lesion size was markedly increased, with increased expression of vascular cell adhesion molecule-1 (VCAM-1) and mononuclear adhesion assessed in vivo [59]. Importantly, insulin sensitivity, glucose tolerance, plasma lipids and blood pressure were unaffected compared to control animals, demonstrating that loss of insulin signaling in the vascular endothelium exacerbates atherosclerosis [59]. Taken together, these data support the hypothesis that insulin does indeed act to suppress atherogenesis and particularly reduces the severity of lesions. 


\section{Potential role of C-peptide on cardiovas cular health}

Proinsulin is cleaved in pancreatic $\beta$-cells to form insulin and C-peptide, which are subsequently secreted in equimolar concentrations. Although C-peptide was initially considered to have no biological function, a substantial number of reports now indicate that C-peptide has actions on the cardiovascular system. Indeed, C-peptide has been proposed to have beneficial effects, such as the stimulation of endothelial NO as well as detrimental, pro-inflammatory effects on the cardiovascular system, although these effects remain controversial [60]. It remains feasible, however, that direct actions of C-peptide may occur alongside insulin, although further research, particularly the identification of the as yet uncharacterized receptor for C-peptide, is required.

\section{Effect of hyperinsulinemia on vascular function}

In cultured vascular cells, the term hyperinsulinemia has been used to describe culture in elevated insulin concentrations for time scales ranging from hours to weeks, whereas in vivo hyperinsulinemia can manifest for far longer. Despite this, prolonged stimulation of cultured endothelial cells with insulin has been reported to suppress PI3K/Akt/eNOS signaling, increase expression of ICAM-1 and VCAM-1 expression in an ERK1/2 or p38MAPK-dependent manner and increase adhesion of promonocytic cells to cultured endothelial cells $[61,62]$. This may reflect engagement of IGF-1 receptors by pathophysiological rather than physiological concentrations of insulin [61].

Compensatory hyperinsulinemia is a common feature of insulin resistance prior to $\beta$-cell failure and can be observed prior to the onset of frank diabetes. Intriguingly, experimental hyperinsulinemia for as little as 3 days has been demonstrated to cause insulin resistance in muscle, indicating that hyperinsulinemia itself can also cause insulin resistance [4]. Separating a specific effect of hyperinsulinemia on vascular function is therefore often complicated in 
clinical studies by insulin resistance and/or metabolic effects of the administered insulin. Hyperinsulinemia has long been considered an independent risk factor for cardiovascular disease and fasting plasma insulin concentrations have been reported to have an inverse relationship with flow-mediated dilation in healthy volunteers [63]. However, recent studies in atherosclerosis-prone mice haploinsufficient with respect to the IR have questioned this relationship. The haploinsufficient IR mice exhibited hyperinsulinemia without altered insulin sensitivity, yet atherosclerotic lesion size was unaffected [64]. Although the majority of the evidence suggests that unlike acute insulin stimulation, hyperinsulinemia may have direct effects on vascular cells that are detrimental to vascular health, the effects of hyperinsulinemia remain uncertain and require further study.

\section{Does vascular insulin resistance contribute to the cardiovascular complications of diabetes?}

O besity, dyslipidemia and pro-inflammatory signaling are central factors that contribute to the development of insulin resistance in classical insulin-sensitive tissues such as muscle, adipose and the liver $[1,2]$. At the molecular level, insulin resistance is thought to be due to metabolites of ectopic lipid stimulating protein kinase C (PKC) and/or proinflammatory cytokines and endoplasmic reticulum stress stimulating c-J un N-terminal kinase (J NK) and inhibitor of NF 8B kinase ${ }^{2}\left(\mathrm{IKK}^{2}\right)$. Activated PKC, J NK and IKK² phosphorylate IRS-1 on inhibitory serine residues, inhibiting activating phosphorylation by the IR on tyrosine residues. As a consequence, the IRS/PI3K/Akt signaling pathway is specifically downregulated in insulin resistance [65] (Figure 3).

It is clear that insulin resistant individuals also exhibit insulin resistance in their cardiovascular tissues. Cardiac tissues from obese rodents or animals fed a high fat diet exhibit impaired insulin-stimulated glucose oxidation and increased fatty acid oxidation $[66,67]$. Furthermore, increased translocation (a measure of activity) of PKC $\alpha$ and increased inhibitory 
IRS-1 serine phosphorylation was observed in cardiac tissue from animals fed a high fat diet [67]. This is likely to impair the ability of the heart to adapt to changes in substrate availability.

Insulin resistance has been observed in blood vessels, in which impaired insulinstimulated vasodilation and NO production is evident $[4,7]$. In cultured endothelial cells, impaired insulin-stimulated PI3K/AKt/NO synthesis has been reported in response to free fatty acids and pro-inflammatory signals including angiotensin II, TNF $\alpha$ and IL6 $[25,68,69,70,71]$. Insulin-stimulated relaxation is impaired in rodent models of type 2 diabetes or obesity $[38,72]$ and can be enhanced by PKC inhibitors [73].

Importantly, a selective insulin resistance has been observed in endothelial cells from volunteers with type 2 diabetes, whereby the insulin-stimulated PI3K/Akt pathway is impaired, yet insulin-stimulated ERK1/2 is unaffected or increased in the endothelium $[7,74]$. Given the different and opposing effects of these insulin signaling pathways on endothelial function, insulin resistance would be predicted to cause endothelial dysfunction, through reduced bioavailability of NO and unaffected or increased ET-1 synthesis. Although a number of studies utilizing mouse models with endothelium-specific downregulation or ablation of insulin signaling have reported reduced NO bioavailability, increased ROS, endothelial dysfunction and enhanced atherogenesis $[22,34,59]$, the models in question have manipulated the activity at the level of the IR, such that both PI3K/Akt and ERKI/2 pathways would be expected to be impaired, unlike the endothelial insulin resistance observed in endothelial cells from patients with type 2 diabetes [74]. In addition, endothelium-specific downregulation of insulin signaling intermediates downstream of the IR would suffer from a lack of specificity as PI3K and Akt are common signaling pathway components for a broad range of stimuli. VSMCs isolated from rodent models of type 2 diabetes also exhibit impaired insulin-stimulated Akt activation $[75,76]$, yet the relevance of VSMC insulin resistance is barely characterized with respect to vascular function. It remains uncertain; therefore, to what extent vascular insulin resistance alone contributes to macrovascular or microvascular complications of diabetes although the weight of evidence 
certainly suggests that insulin does have clinically-relevant actions on blood vessels that act to suppress atherosclerosis.

\section{Direct vascular actions of antidiabetic pharmaceuticals}

Intensive diabetes treatment with insulin was reported to substantially lower the risk of cardiovascular disease in people with type 1 diabetes, indicating that insulin has beneficial effects on the cardiovascular system [77]. Intriguingly, it has been recently reported that therapy with insulin glargine for 6 years had no effect on cardiovascular outcomes in volunteers with type 2 diabetes, impaired glucose tolerance or impaired fasting glucose, compared to standard treatment [78]. This implies that insulin has no beneficial cardiovascular effect in an insulinresistant group, although it should be noted that this study was not testing intensive insulin treatment, rather the use of insulin compared to standard care in a glycemia-controlled manner, in volunteers many of whom were using other oral hypoglycaemic agents. Indeed, a number of pharmacological agents in current use for the treatment of patients with type 2 diabetes have been reported to have direct vascular effects independent of their capacity to improve glycemia. Metformin suppresses hepatic gluconeogenesis, thereby normalizing glycemia, yet metformin has also been reported to reduce the incidence of myocardial infarction and stroke in comparison with sulfonylurea or insulin causing matched improvement in glycemic control [79]. Furthermore, metformin therapy has been reported to decrease myocardial injury and improve left ventricular function and survival after ischemia and reperfusion [79]. These potential direct actions of metformin on vascular tissues are thought to be mediated, at least in part, by activation of AMP-activated protein kinase (AMPK) $[79,80]$. AMPK mimics insulin's action in cardiovascular tissues by phosphorylation and activation of eNOS, stimulating NO synthesis in addition to NO-dependent and NO-independent suppression of endothelial pro-inflammatory signaling $[81,82]$. 
The thiazolidinedione (TZD) class of drugs improve insulin sensitivity by virtue of their stimulation of peroxisome proliferator-activated receptor- $\gamma(\operatorname{PPAR} \gamma)$, which regulates the transcription of metabolic genes [83]. As with metformin, TZDs have been reported to rapidly stimulate NO synthesis via AMPK-stimulated phosphorylation of eNOS in cultured endothelial cells and also suppress pro-inflammatory signaling $[81,83]$.

Incretin based therapies such as glucagon-like peptide-1 (GLP-1) receptor agonists and dipeptydil-peptidase-4 (DPP-4) inhibitors are used in patients with type 2 diabetes to upregulate GLP-1 signaling leading to increased insulin secretion in response to a meal $[84,85]$. It is becoming apparent that these drugs also have direct effects on myocardial and endothelial cells including stimulation of NO and suppression of proinflammatory signaling [84,85].

As all of these antidiabetic agents may directly mimic the effects of insulin in the endothelium without altering insulin signaling whilst improving the metabolic consequences of diabetes, understanding the molecular mechanisms by which they act is of the utmost importance.

\section{Concluding remarks}

In summary, insulin has direct effects on cardiomyocytes, endothelial cells, VSMCs, erythrocytes, leukocytes and platelets in vitro and there is substantial evidence that the effects of insulin on blood vessels contribute directly to the maintenance of cardiovascular health. Evidence from studies using animal models with tissue-specific downregulation of insulin signaling has been instrumental in demonstrating that insulin regulates substrate utilization in the heart, endothelial NO synthesis and has an anti-inflammatory, atheroprotective action in vivo. Some care should be taken when interpreting the data using these genetic models, however, as they may not accurately reflect defects in insulin signaling observed in insulin resistance. A host of factors including hyperglycemia, dyslipidemia, increased proinflammatory signaling and cellular reactive oxygen species are all associated with cardiovascular disease 
and are observed in insulin resistance or diabetes such that examining the role of insulin in the regulation of cardiovascular health in man is complicated by systemic effects of the administered insulin. Furthermore, sustained elevated concentrations of insulin such as those observed in hyperinsulinemia may be detrimental to cardiovascular health, suggesting the duration of signal is important in the action of insulin. Further characterisation of the role of insulin in blood vessels, the signaling pathways engaged by insulin and their modulation by factors altered in diabetes may identify novel therapeutic targets to limit or prevent the vascular complications of diabetes. 


\section{FUTURE PERSPECTIVE}

Although the actions of insulin in blood vessels and the heart have been partially characterized, significant questions remain that require resolution. Understanding the molecular mechanisms by which insulin acts in cardiovascular tissues is vital for the development of appropriate therapeutic targets for the normalization of vascular insulin resistance. Insulin infusion is used in the clinical setting to improve outcomes in acute coronary patients, yet there is still considerable debate as to its efficacy. To address this, a large multicentre randomized clinical trial would be exceedingly useful to confirm the protective effects of insulin on the cardiovascular system. Given the potential for differential effects of insulin depending on the duration of stimulation, future studies should concentrate on investigating the role of hyperinsulinemia on cardiovascular function. The endothelium is perhaps the best characterized to date of the cardiovascular tissues with respect to insulin action. In the future, it would be desirable to greatly improve our understanding of direct actions of insulin in vascular smooth muscle, leukocytes and PVAT, in which there is a particular dearth of information at present. Even in the endothelium, it is unclear how insulin stimulates eNOS as it is clear that phosphorylation is not the only mechanism by which eNOS is regulated. Future research examining the effect of insulin on eNOS trafficking, dimerisation and association with regulatory proteins is required to address this, as is research to assess the influence of ADMA in the action of insulin in healthy or insulin-resistant vessels. Inducible, tissue-specific downregulation of insulin signaling will would improve our understanding of what happens to the cardiovascular tissues when their insulin resistance becomes impaired in adulthood rather than being impaired for the entirety of development. Furthermore, it is now clear that existing antidiabetic therapies have direct cardiovascular actions independent of their effects on metabolism. The thorough investigation of the mechanisms by which these cardiovascular actions are manifest is warranted to identify useful molecular targets for the design of future therapies. 


\section{EXECUTIVE SUMMARY}

\section{Vascular effects of insulin}

- Insulin has direct effects on blood vessels, independent of effects on metabolism

- Insulin stimulates vasodilation, capillary recruitment and improves nutrient delivery to muscle.

\section{Cardiac actions of insulin}

- Insulin stimulates glucose oxidation and attenuates fatty acid oxidation, such that appropriate insulin signaling is required to maintain flexibility during changes in energy substrate supply.

\section{Endothelial effects of insulin}

- Insulin stimulates synthesis of the vasodilator NO at least in part by Akt-mediated phosphorylation and activation of endothelial nitric oxide synthase.

- Insulin stimulates synthesis of the vasoconstrictor endothelin-1 by an ERK1/2-dependent mechanism.

- Insulin has anti-inflammatory actions that limit atherogenesis and stimulate endothelial cell proliferation.

\section{VSMC effects of insulin}

- Insulin regulates vascular tone and VSMC proliferation by endothelium-dependent and direct endothelium-independent actions.

\section{Anti-atherogenic actions of insulin}

- Insulin reduces atherogenesis, likely to be a consequence of reduced NO synthesis and NO-independent mechanisms that reduce pro-inflammatory signaling and limit leukocyte adhesion to blood vessels.

- The direct effects of insulin on leukocytes are poorly characterized.

\section{Vascular insulin resistance}


- Cardiovascular diseases are the leading cause of morbidity and mortality in people with insulin resistant type 2 diabetes

- Obesity, dyslipidemia and pro-inflammatory signaling are central factors that contribute to the development of insulin resistance. These activate protein kinases that inhibit insulin signaling through the IRS/PI3K/Akt signaling pathway without altering ERKI/2 activation.

- The balance of NO and endothelin-1 synthesis is important in the regulation of vascular tone and is altered in insulin resistance when NO synthesis is specifically impaired.

- Hyperinsulinemia, present in insulin-resistant individuals prior to the development of diabetes may reduce NO bioavailability and promote atherogenesis, suggesting insulin has rapid beneficial effects on vascular health yet becomes detrimental upon long term stimulation.

\section{Future perspective}

- Insulin stimulation of IRS/PI3K/Akt signaling is specifically downregulated in insulin resistance. Substantial insights into the role of insulin in cardiovascular tissues have been obtained using rodents genetically manipulated to downregulate insulin signaling in specific tissues. It is still unclear, however, to what degree hyperinsulinemia is responsible for impaired cardiovascular function. Furthermore, future studies need to concentrate on further characterizing the role of insulin in vascular smooth muscle, vascular leukocytes and perivascular adipose tissue, which are currently underinvestigated. 


\section{ABBREVIATIONS}

Akt; protein kinase $B$; AMPK; AMP-activated protein kinase; APS, adaptor protein with pleckstrin homology and $\mathrm{Src}$ homology 2 domains; $\mathrm{BH}_{4}$, reduced tetrahydrobiopterin; $\mathrm{CAP}, \mathrm{C}-$ Cbl-associated protein; eNOS, endothelial NO synthase; ET-1, endothelin-1; GLP-1, glucagonlike peptide-1; Grb2, growth factor receptor-bound protein 2; ICAM-1, intercellular adhesion molecule-1; IGF-1, insulin-like growth factor-1; IGFR, insulin-like growth factor receptor; IKK², inhibitor of NF8B kinase ${ }^{2}$; IL6, interleukin-6; IR, insulin receptor; IRS, insulin receptor substrate; J NK, J un N-terminal kinase; LDL, low density lipoprotein; MCP-1, monocyte chemoattractant protein-1; mTORC2, mammalian target of rapamycin complex $2 ; \mathrm{NF} \kappa \mathrm{B}$, nuclear factor $\kappa B$; NO, nitric oxide; PDK1, phosphoinositide-dependent kinase 1; PI3K, phosphatidylinositol 3'-kinase; $\mathrm{PIP}_{3}$, phosphatidylinositol-3,4,5-trisphosphate; PKC, protein kinase C; PKG, CGMP-dependent protein kinase; PPAR $\gamma$, proliferator-activated receptor- $\gamma$; PVAT, perivascular adipose tissue; ROS, reactive oxygen species; Shc, Src-homology and collagen-like protein; SOS, Son of Sevenless; TNF $\alpha$, tumor necrosis factor- $\alpha$; TZD, thiazolidinedione; VCAM-1, vascular cell adhesion molecule-1; VSMC, vascular smooth muscle cell. 


\section{REFERENCES}

1. Rask-Madsen C, Kahn R. Tissue-specific insulin signalling, metabolic syndrome, and cardiovascular disease. Arterioscler. Thromb. Vasc. Biol. 32(9), 2052-2059 (2012). * excellent review of tissue-specific insulin resistance

2. Glass CK, Olefsky J M. Inflammation and lipid signaling in the etiology of insulin resistance. Cell Metab. 15(5), 635-645 (2012).

3. $\mathrm{Xu} \mathrm{J,} \mathrm{Zou} \mathrm{MH.} \mathrm{Molecular} \mathrm{insights} \mathrm{and} \mathrm{therapeutic} \mathrm{targets} \mathrm{for} \mathrm{diabetic} \mathrm{endothelial}$ dysfunction. Circulation 120(13), 1266-1286 (2009)

4. DeFronzo RA. Insulin resistance, lipotoxicity, type 2 diabetes and atherosclerosis: the missing links. Diabetologia 53(7), 1270-1287 (2010).

5. Leto D, Saltiel AR. Regulation of glucose transport by insulin: traffic control of GLUT4. Nat. Rev. Cell Biol. 13(6), 383-396 (2012).

6. Siddle K. Molecular basis of signaling specificity of insulin and IGF receptors: neglected corners and recent advances. Front. Endocrinol. 3, 34 (2012).

7. Potenza MA, Addabbo F, Montagnani M. Vascular actions of insulin with implications for endothelial dysfunction. Am. J. Physiol. Endocrinol. Metab. 297(3), E568-E577 (2009).* excellent review focusing on the molecular actions of insulin in the endothelium

8. Steinberg HO, Brechtel G, J ohnson A, Fineberg N, Baron AD. Insulin-mediated skeletal muscle vasodilation is nitric oxide dependent. A novel action of insulin to increase nitric oxide release. J. Clin. Invest. 94, 1172-1174 (1994).* first demonstration of insulinstimulated, NO-dependent vasodilation in humans.

9. Scherrer U, Randin D, Vollenweider P, Vollenweider L, Nicod P. Nitric oxide release accounts for insulin's vascular effects in humans. J . Clin. Invest. 94(6), 2511-2515 (1994).

* first demonstration of insulin-stimulated, NO-dependent vasodilation in humans. 
10. Barrett EJ, Eggleston EM, Inyard AC, et al. The vascular actions of insulin control its delivery to muscle and regulate the rate-limiting step in skeletal muscle insulin action. Diabetologia 52(5), 752-764 (2009).

11. Abel ED, O'Shea KM, Ramasamy R. Insulin resistance: metabolic mechanisms and consequences in the heart. Arterioscler. Thromb. Vasc. Biol. 32(9), 2068-2076 (2012).

12. Boudina S, Bugger $\mathrm{H}$, Sena $\mathrm{S}$, et al. Contribution of impaired myocardial insulin signaling to mitochondrial dysfunction and oxidative stress in the heart. Circulation 119(9), 12721283 (2009). **examination of cardiac-specific roles of insulin using a tissue-specific

\section{IR knockout mouse.}

13. Liu J, Jahn LA, Fowler DE, Barrett EJ, Cao W, Liu Z. Free fatty acids induce insulin resistance in both cardiac and skeletal muscle microvasculature in humans. J. Clin. Endocrinol. Metab. 96(2), 438-446 (2011).

14. von Lewinski D, Rainer PP, Gasser R, et al. Glucose-transporter-mediated positive inotropic effects in human myocardium of diabetic and nondiabetic patients. Metabolism 59(7), 1020-1028 (2010).

15. Ng KW, Allen ML, Desai A, Macrae D, Pathan N. Cardioprotective effects of insulin: how intensive insulin therapy may benefit cardiac surgery patients. Circulation 125(5), 721-728 (2012).

16. Ellsworth ML, Ellis CG, Goldman D, Stephenson AH, Dietrich HH, Sprague RS. Erythrocytes: oxygen sensors and modulators of vascular tone. Physiology, 107-116 (2009).

17. Hanson MS, Ellsworth ML, Achilleus $D$, et al. Insulin inhibits low oxygen-induced ATP release from human erythrocytes: implication for vascular control. Microcirculation 16(5), 424-433 (2009). 
18. Randriamboavonjy V, Schrader J, Busse R, Fleming I. Insulin induces the release of vasodilator compounds from platelets by a nitric oxide-G kinase-VAMP-3-dependent pathway. J . Exp. Med. 199(3), 347-356 (2004).

19. Worthley MI, Holmes AS, Willoughby SR, et al. The deleterious effects of hyperglycemia on platelet function in diabetic patients with acute coronary syndromes mediation by superoxide production, resolution with intensive insulin administration. J. Am. Coll. Cardiol. 49(3), 304-310 (2007).

20. Kobayashi T, Nogami T, Taguchi K, Matsumoto T, Kamata K. Diabetic state, high plasma insulin and angiotensin II combine to augment endothelin-1-induced vasoconstriction via ETA receptors and ERK. Br. J. Pharmacol. 155(7), 974-983 (2008).

21. Michel T, Vanhoutte PM. Cellular signalling and NO production. Pflugers Arch. 459(6), 807-816 (2010).

22. Abbas $\mathrm{A}$, Imrie $\mathrm{H}$, Viswambharan $\mathrm{H}$, et al. The insulin-like growth factor-1 receptor is a negative regulator of nitric oxide bioavailability and insulin sensitivity in the endothelium. Diabetes 60(8), 2169-2178 (2011). **examination of endothelial-specific role of IGF-1.

23. Imrie $H$, Viswambharan $H$, Sukumar $P$ et al. Novel Role of the IGF-1 Receptor in Endothelial Function and Repair: Studies in Endothelium-Targeted IGF-1 Receptor Transgenic Mice. Diabetes 61(9), 2359-2368 (2012). **examination of endothelialspecific role of IGF-1.

24. Mount PF, Kemp BE, Power DA. Regulation of endothelial and myocardial NO synthesis by multi-site eNOS phosphorylation. J. Mol. Cell. Cardiol. 42(2), 271-279 (2007).

25. Ritchie SA, Kohlhaas CF, B oyd AR, et al. Insulin-stimulated phosphorylation of endothelial nitric oxide synthase at Ser615 contributes to nitric oxide synthesis. Biochem. J . 426(1), 85-90 (2010). 
26. Kohlhaas CF, Morrow VA, J hakra N, et al. Insulin rapidly stimulates L-arginine transport in human aortic endothelial cells via Akt. Biochem. Biophys. Res. Commun. 412(4), 747-751 (2011).

27. Fleming I. Molecular mechanisms underlying the activation of eNOS. Eur. J. Physiol. 459(6), 793-806 (2010). * excellent review of eNOS regulation

28. Wang H, Wang AX, Liu Z, Chai W, Barrett EJ. The trafficking/interaction of eNOS and caveolin-1 induced by insulin modulates endothelial nitric oxide production. Mol. Endocrinol. 23(10), 1613-1623 (2009). *describes phosphorylation-independent

\section{actions of insulin on eNOS}

29. Zhang QJ, Holland WL, Wilson L, et al. Ceramide mediates vascular dysfunction in dietinduced obesity by PP2A-mediated dephosphorylation of the eNOS-Akt complex. Diabetes 61(7), 1848-1859 (2012).

30. González M, Gallardo V, Rodríguez N, et al. Insulin-stimulated L-arginine transport requires SLC7A1 gene expression and is associated with human umbilical vein relaxation. J. Cell. Physiol. 226(11), 2916-2924 (2011).

31. Anderssohn M, Schwedhelm E, Lüneburg N, Vasan RS, Böger RH. Assymetric dimethylarginine as a mediator of vascular dysfunction and a marker of cardiovascular disease and mortality: an intriguing interaction with diabetes mellitus. Diab. Vasc. Dis. Res. 7(2), 105-118 (2010).

32. Cunnington C, Channon KM. Tetrahydrobiopterin: pleiotropic roles in cardiovascular pathophysiology. Heart 96(23), 1872-1877 (2010).

33. Ji L, Fu F, Zhang L, et al. Insulin attenuates myocardial ischemia/reperfusion injury via reducing oxidative/nitrative stress. Am. J. Physiol. Endocrinol. Metab. 298(4), E871-E880 (2010). 
34. Duncan ER, Crossey PA, Walker S, et al. Effect of endothelium-specific insulin resistance on endothelial function in vivo. Diabetes $57(12), 3307-3314$ (2008). **examination of endothelial-specific insulin resistance using mouse model.

35. Ishii M, Shimizu S, Nagai T, Shiota K, Kiuchi Y, Yamamoto T. Stimulation of tetrahydrobiopterin synthesis induced by insulin: possible involvement of phosphatidylinositol 3-kinase. Int. J . Biochem. Cell Biol. 33(1), 65-73 (2001).

36. Haines RJ, Corbin KD, Pendleton LC, Meininger CJ, Eichler DC. Insulin transcriptionally regulates argininosuccinate synthase to maintain vascular endothelial function. Biochem. Biophys. Res. Commun. 421(1), 9-14 (2012).

37. Vicent $\mathrm{D}$, llany $\mathrm{J}, \mathrm{Kondo} \mathrm{T}$, et al. The role of endothelial insulin signaling in the regulation of vascular tone and insulin resistance. J. Clin. Invest. 111(9), 1373-1380 (2003).

38. Fisslthaler B, Loot AE, Mohamed A, Busse R, Fleming I. Inhibition of endothelial nitric oxide synthase activity by proline-rich tyrosine kinase 2 in response to fluid shear stress and insulin. Circ. Res. 102(12), 1520-1528 (2008).

39. Mazzuca MQ, Khalil RA. Vascular endothelin receptor type B: structure, function and dysregulation in vascular disease. Biochem. Pharmacol. 84(2), 147-162 (2012).

40. Wang Y, Cheng KK, Lam KS, et al. APPL1 counteracts obesity-induced vascular insulin resistance and endothelial dysfunction by modulating the endothelial production of nitric oxide and endothelin-1 in mice. Diabetes (11), 3044-3054 (2011).

41. Kapakos G, Bouallegue A, Daou GB, Srivastava AK. Modulatory Role of Nitric Oxide/cGMP System in Endothelin-1-Induced Signaling Responses in Vascular Smooth Muscle Cells. Curr. Cardiol. Rev. 6(4), 247-254 (2010).

42. Liu Y, Petreaca M, Martins-Green M. Cell and molecular mechanisms of insulin-induced angiogenesis. J. Cell. Mol. Med. 13(11-12), 4492-4504 (2009). 
43. Waldow T, Witt W, Weber E, Matschke K. Nitric oxide donor-induced persistent inhibition of cell adhesion protein expression and NFkappaB activation in endothelial cells. Nitric Oxide 15(2), 103-113 (2006).

44. Dandona P, Chaudhuri A, Ghanim H, Mohanty P. Insulin as an anti-inflammatory and antiatherogenic modulator. J. Am. Coll. Cardiol. 53(5 Suppl), S14-S20 (2009).

45. Wang H, Wang AX, Liu Z, Barrett EJ. Insulin signaling stimulates insulin transport by bovine aortic endothelial cells. Diabetes 57(3), 540-547 (2008).

46. Chiu J D, Richey J M, Harrison LN, et al. Direct administration of insulin into skeletal muscle reveals that the transport of insulin across the capillary endothelium limits the time course of insulin to activate glucose disposal. Diabetes 57(4), 828-835 (2008).

47. Muniyappa R, Montagnani M, Koh KK, Quon MJ. Cardiovascular actions of insulin. Endocr. Rev. 28(5), 463-491 (2007).

48. Lee JH, Ragolia L. AKT phosphorylation is essential for insulin-induced relaxation of rat vascular smooth muscle cells. Am. J. Physiol. Cell. Physiol. 291(6), C1355-C1365 (2006).

49. Lee JH, Palaia T, Ragolia L. Impaired insulin-stimulated myosin phosphatase Rhointeracting protein signaling in diabetic Goto-Kakizaki vascular smooth muscle cells. Am. J . Physiol. Cell. Physiol. 302(9), C1371-C1381 (2012).

50. Isenovic ER, Kedees MH, Haidara MA, Trpkovic A, Mikhailidis DP, Marche P. Involvement of ERK1/2 kinase in insulin-and thrombin-stimulated vascular smooth muscle cell proliferation. Angiology 61(4), 357-364 (2010).

51. Lightell DJ J r, Moss SC, Woods TC. Loss of canonical insulin signaling accelerates vascular smooth muscle cell proliferation and migration through changes in p27Kip1 regulation. Endocrinology 152(2), 651-658 (2011).

52. Liang $\mathrm{CP}$, Han S, Senokuchi T, Tall AR. The macrophage at the crossroads of insulin resistance and atherosclerosis. Circ. Res. 100(11), 1546-1555 (2007). 
53. Mita T, Azuma K, Goto $\mathrm{H}$, et al. IRS-2 deficiency in macrophages promotes their accumulation in the vascular wall. Biochem. Biophys. Res. Commun. 415(4), 545-550 (2011).

54. Eringa EC, Bakker W, van Hinsbergh VW. Paracrine regulation of vascular tone, inflammation and insulin sensitivity by perivascular adipose tissue. Vascul .Pharmacol. 56(5-6), 204-209 (2012). *review of role of perivascular adipose tissue in vascular regulation.

55. Greenstein AS, Khavandi K, Withers SB, et al. Local inflammation and hypoxia abolish the protective anticontractile properties of perivascular fat in obese patients. Circulation 119(12), 1661-1670 (2009).

56. Breen DM, Chan KK, Dhaliwall J K, et al. Insulin increases reendothelialization and inhibits cell migration and neointimal growth after arterial injury. Arterioscler. Thromb. Vasc. Biol. 29(7), 1060-1066 (2009).

57. Han S, Liang CP, DeVries-Seimon $T$, et al. Macrophage insulin receptor deficiency increases ER stress-induced apoptosis and necrotic core formation in advanced atherosclerotic lesions. Cell Metab. 3(4), 257-266 (2006). ${ }^{* *}$ demonstration of antiatherogenic action of insulin in macrophages

58. Baumgartl J, Baudler S, Scherner M, et al. Myeloid lineage cell-restricted insulin resistance protects apolipoproteinE-deficient mice against atherosclerosis. Cell Metab. 3(4), 247-256 (2006).

59. Rask-Madsen C, Li Q, Freund B, et al. Loss of insulin signaling in vascular endothelial cells accelerates atherosclerosis in apolipoprotein E null mice. Cell Metab. 11(5), 379-389 (2010). **demonstration of anti-atherogenic action of insulin in endothelium

60. Vasic D, Walcher D. C-peptide: a new mediator of atherosclerosis in diabetes. Mediators. Inflamm. 2012, 858692. (2012) 
61. Madonna R, De Caterina R. Prolonged exposure to high insulin impairs the endothelial PI3-kinase/Akt/nitric oxide signalling. Thromb. Haemost. 101(2), 345-350 (2009).

62. Li G, Barrett EJ, Ko SH, Cao W, Liu Z. Insulin and insulin-like growth factor-I receptors differentially mediate insulin-stimulated adhesion molecule production by endothelial cells. Endocrinology 150(8), 3475-3482 (2009).

63. Ardigo D, Franzini L, Valtuena S, Monti LD, Reaven GM, Zavaroni I. Relation of plasma insulin levels to forearm flow-mediated dilatation in healthy volunteers. Am. J. Cardiol. 97(8), 1250-1254 (2006). *demonstrates association between plasma insulin concentrations and blood flow.

64. Rask-Madsen C, Buonomo E, Li Q, et al. Hyperinsulinemia does not change atherosclerosis development in apolipoprotein E null mice. Arterioscler. Thromb. Vasc. Biol. 32(5), 1124-1131 (2012). **evidence that hyperinsulinemia has little effect on atherosclerosis.

65. Copps KD, White MF. Regulation of insulin sensitivity by serine/threonine phosphorylation of insulin receptor substrate proteins IRS1 and IRS2. Diabetologia 55(10), 2565-2582 (2012).

66. Tabbi-Anneni I, Buchanan J, Cooksey RC, Abel ED. Captopril normalizes insulin signaling and insulin-regulated substrate metabolism in obese (ob/ob) mouse hearts. Endocrinology 149(8), 4043-4050 (2008).

67. Zhang L, Ussher JR, Oka T, Cadete VJ, Wagg C, Lopaschuk GD. Cardiac diacylglycerol accumulation in high fat-fed mice is associated with impaired insulin-stimulated glucose oxidation. Cardiovasc. Res. 89(1), 148-156 (2011).

68. Kim J A, Jang HJ, Martinez-Lemus LA, Sowers J R. Activation of mTOR/p70S6 kinase by ANG II inhibits insulin-stimulated endothelial nitric oxide synthase and vasodilation. Am. J . Physiol. Endocrinol. Metab. 302(2), E201-E208 (2012). 
69. Andreozzi F, Laratta E, Procopio $C$, et al. Interleukin-6 impairs the insulin signaling pathway, promoting production of nitric oxide in human umbilical vein endothelial cells. Mol. Cell. Biol. 27(6), 2372-2383 (2007).

70. Li G, Barrett EJ, Barrett MO, Cao W, Liu Z. Tumor necrosis factor-alpha induces insulin resistance in endothelial cells via a p38 mitogen-activated protein kinase-dependent pathway. J . Biol. Chem. 284(25), 17120-17128 (2009).

71. J ung $\mathrm{CH}$, Lee WJ, Hwang J Y, et al. Vaspin protects vascular endothelial cells against free fatty acid-induced apoptosis through a phosphatidylinositol 3-kinase/Akt pathway. Biochem. Biophys. Res. Commun. 413(2), 264-269 (2011).

72. Nemoto S, Kobayashi T, Taguchi K, Matsumoto T, Kamata K. Losartan improves aortic endothelium-dependent relaxation via proline-rich tyrosine kinase $2 / \mathrm{Src} / \mathrm{Akt}$ pathway in type 2 diabetic Goto-Kakizaki rats. Am. J. Physiol. Heart. Circ. Physiol. 301(6), H2383H2394 (2011).

73. Taguchi K, Kobayashi T, Matsumoto T, Kamata K. Dysfunction of endothelium-dependent relaxation to insulin via PKC-mediated GRK2/Akt activation in aortas of ob/ob mice. Am. J . Physiol. Heart. Circ. Physiol. 301(2), H571-H583 (2011).

74. Gogg S, S mith U, J ansson PA. Increased MAPK activation and impaired insulin signaling in subcutaneous microvascular endothelial cells in type 2 diabetes: the role of endothelin-

1. Diabetes 58(10), 2238-2245 (2009). **demonstration of selective insulin resistance in human vascular cells.

75. Doronzo G, Russo I, Mattiello L, Riganti C, Anfossi G, Trovati M. Insulin activates hypoxiainducible factor-1alpha in human and rat vascular smooth muscle cells via phosphatidylinositol-3 kinase and mitogen-activated protein kinase pathways: impairment in insulin resistance owing to defects in insulin signalling. Diabetologia 49(5), 1049-1063 (2006). 
76. Lee JH, Palaia T, Ragolia L. Impaired insulin-mediated vasorelaxation in diabetic GotoKakizaki rats is caused by impaired Akt phosphorylation. Am. J. Physiol. Cell. Physiol. 296(2), C327-C 338 (2009).

77. Nathan DM, Cleary PA, Backlund JY, et al; Diabetes Control and Complications Trial/Epidemiology of Diabetes Interventions and Complications (DCCT/EDIC) Study Research Group. Intensive diabetes treatment and cardiovascular disease in patients with type 1 diabetes. N. Engl. J . Med. 353(25), 2643-2653 (2005).

78. Gerstein HC, Bosch J, Dagenais GR, et al;ORIGIN Trial Investigators. Basal insulin and cardiovascular and other outcomes in dysglycemia. N. Engl. J. Med. 367(4), 319-328 (2012).

79. Boyle JG, Salt IP, McKay GA. Metformin action on AMP-activated protein kinase: a translational research approach to understanding a potential new therapeutic target. Diabet. Med. 27(10), 1097-1106 (2010).

80. Wang S, Song P, Zou MH. AMP-activated protein kinase, stress responses and cardiovascular diseases. Clin. Sci. 122(12), 555-573 (2012).

81. Boyle J G, Logan PJ, Ewart MA, et al. Rosiglitazone stimulates nitric oxide synthesis in human aortic endothelial cells via AMP-activated protein kinase. J. Biol. Chem. 283(17), 11210-11217 (2008).

82. Ewart MA, Kohlhaas CF, Salt IP. Inhibition of tumor necrosis factor alpha-stimulated monocyte adhesion to human aortic endothelial cells by AMP-activated protein kinase. Arterioscler. Thromb. Vasc. Biol. 28(12), 2255-2257 (2008).

83. Balakumar $\mathrm{P}$, Kathuria S. Submaximal $\mathrm{PPAR}^{3}$ activation and endothelial dysfunction: new perspectives for the management of cardiovascular disorders. Br. J. Pharmacol. 166(7):1981-1992 (2012). 
84. Forst $\mathrm{T}$, Weber MM, Pfütner A. Cardiovascular benefits of GLP-1-based therapies in patients with diabetes mellitus type 2: effects on endothelial and vascular dysfunction beyond glycemic control. Exp. Diabetes Res. 2012, 635472 (2012).

85. Fadini GP, Avogaro A. Cardiovascular effects of DPP-4 inhibition: beyond GLP-1. Vascul. Pharmacol. 55(1-3), 10-16 (2011).

101. CDC: National Diabetes Fact Sheet 2011. Available at www.cdc.gov/diabetes/pubs/pdf/ndfs 2011.pdf. Accessed August 2012. 


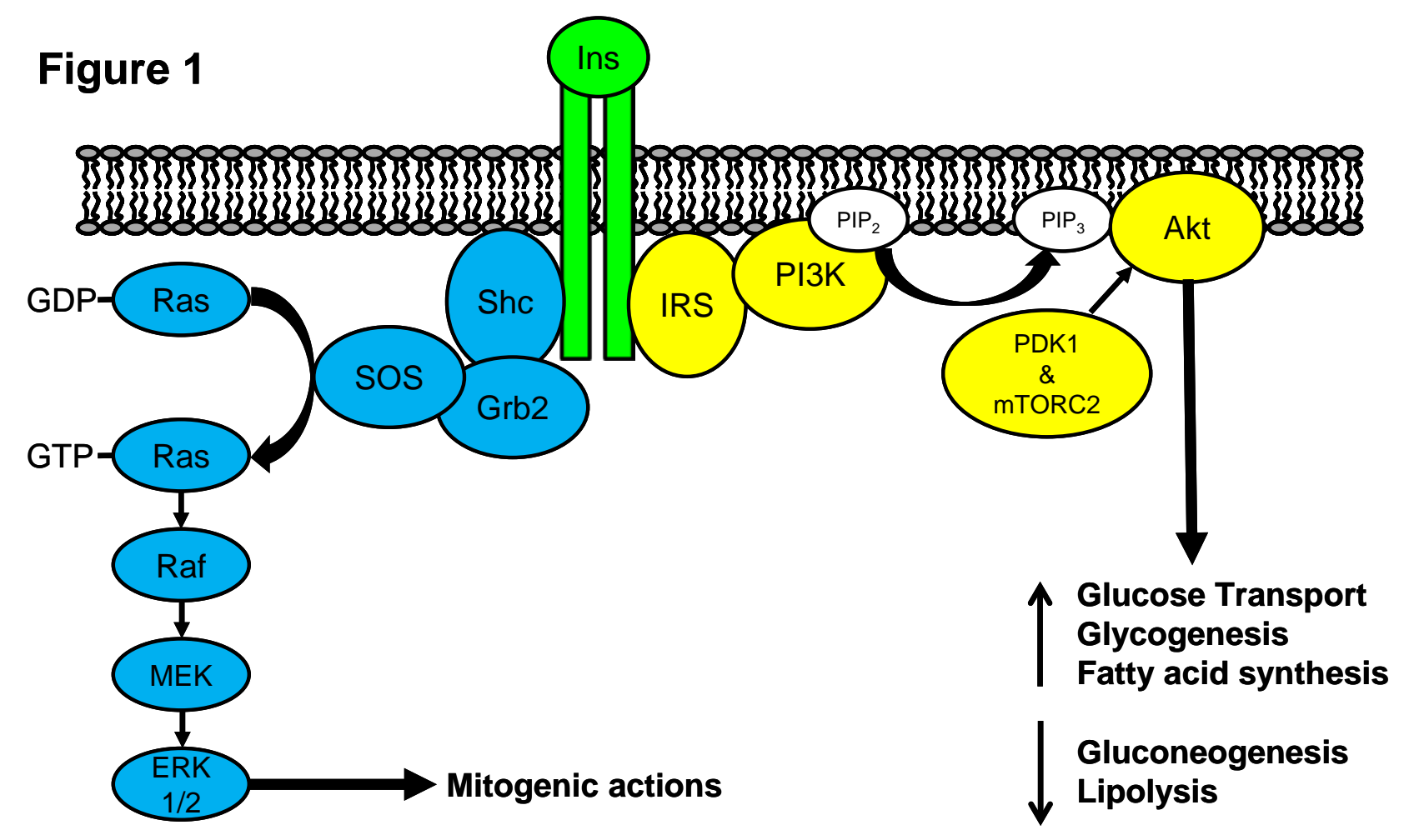

Figure 1: Insulin signaling pathways

Insulin (Ins) binding to the dimeric insulin receptor tyrosine kinase stimulates autophosphorylation leading to binding of the insulin receptor substrate (IRS) and Src-homology and collagen-like (Shc) adapter proteins. Upon binding to the activated insulin receptor, IRSS are phosphorylated, generating docking sites for the recruitment of phosphatidylinositol 3'kinase (PI3K), which generates phosphatidylinositol-3,4,5-trisphosphate ( $\left.\mathrm{PIP}_{3}\right)$ from phosphatidylinositol-4,5-bisphosphate ( $\left.\mathrm{PIP}_{2}\right) . \mathrm{PIP}_{3}$ recruits the serine/threonine protein kinase Akt to the plasma membrane, and Akt is subsequently phosphorylated and activated by phosphoinositide-dependent kinase 1 (PDK1) and mammalian target of rapamycin complex 2 (mTORC2). The IRS-PI3K-Akt pathway is the principal signaling pathway by which insulin exerts the majority of its metabolic effects. Activation of the insulin receptor stimulates binding of growth factor receptor-bound protein 2 (G rb2) to Shc, which subsequently stimulates Son of Sevenless (SOS) to catalyse GDP-GTP exchange on the small G-protein Ras. GTP-bound active Ras initiates the Raf-MEK-ERK1/2 protein kinase cascade, which mediates many of the mitogenic actions of insulin. 


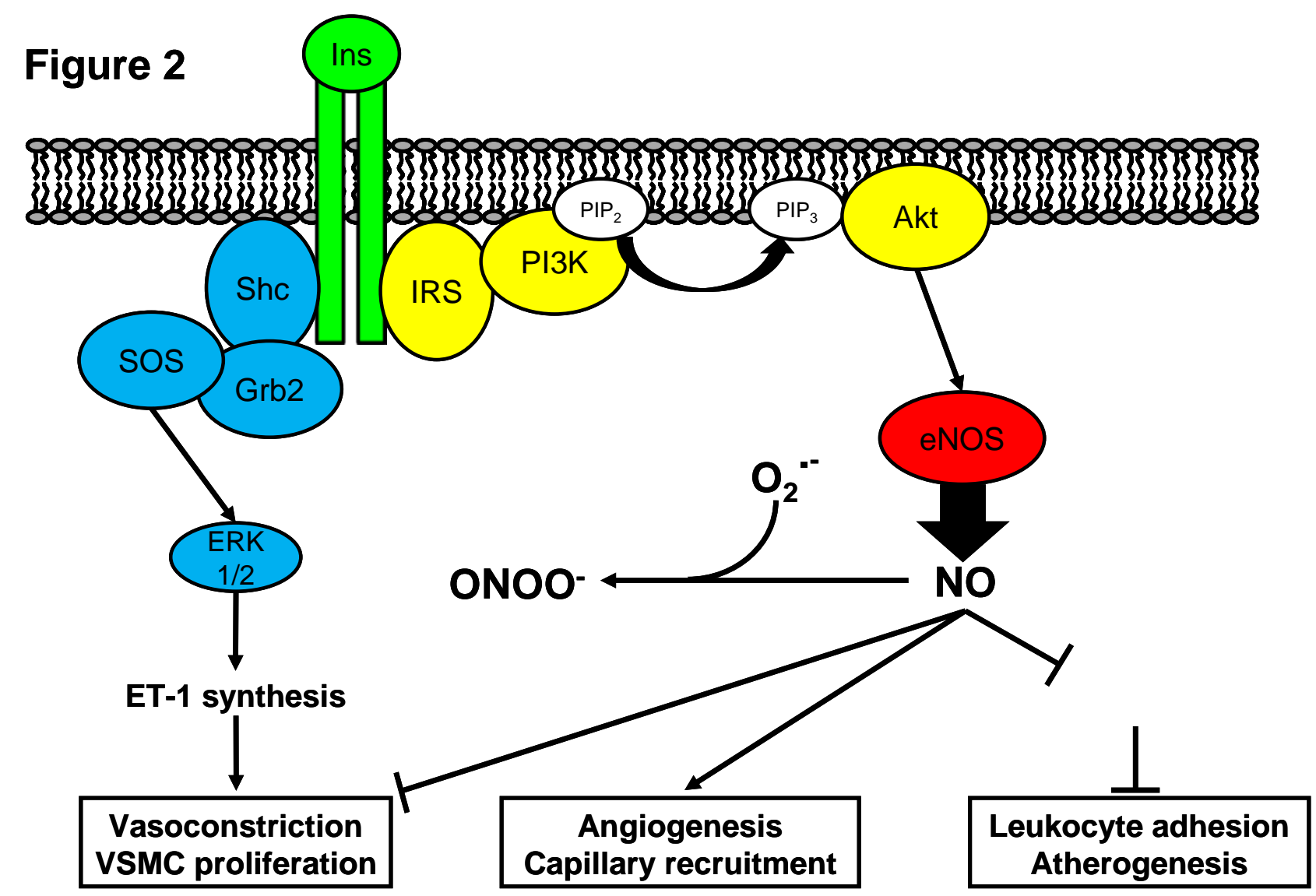

Figure 2: Insulin-stimulated NO and endothelin-1 synthesis

Insulin (Ins) stimulates activation of Akt as described in Figure 1, which phosphorylates and activates endothelial NO synthase (eNOS). The NO synthesized stimulates vasodilation, angiogenesis and capillary recruitment, whilst inhibiting pro-atherogenic vascular smooth muscle cell (VSMC) proliferation, expression of the chemokine monocyte chemoattractant protein-1 (MCP-1) and vascular cell adhesion molecule-1 (VCAM-1). NO is sequestered by elevated concentrations of superoxide, leading to production of highly reactive peroxynitrite. Insulin stimulates the mitogenic protein kinases ERK1/2 through the pathway described in Figure 1, stimulating release of endothelin-1 (ET-1) which stimulates vasodilation and VSMC proliferation. Akt, protein kinase B; Grb2, growth factor receptor-bound protein 2; IRS, insulin receptor substrate; PI3K, phosphatidylinositol 3'-kinase; $\mathrm{PIP}_{2}$, phosphatidylinositol-4,5bisphosphate; PIP 3 , phosphatidylinositol-3,4,5-trisphosphate; Shc, Src-homology and collagenlike; SOS, Son of S evenless. 


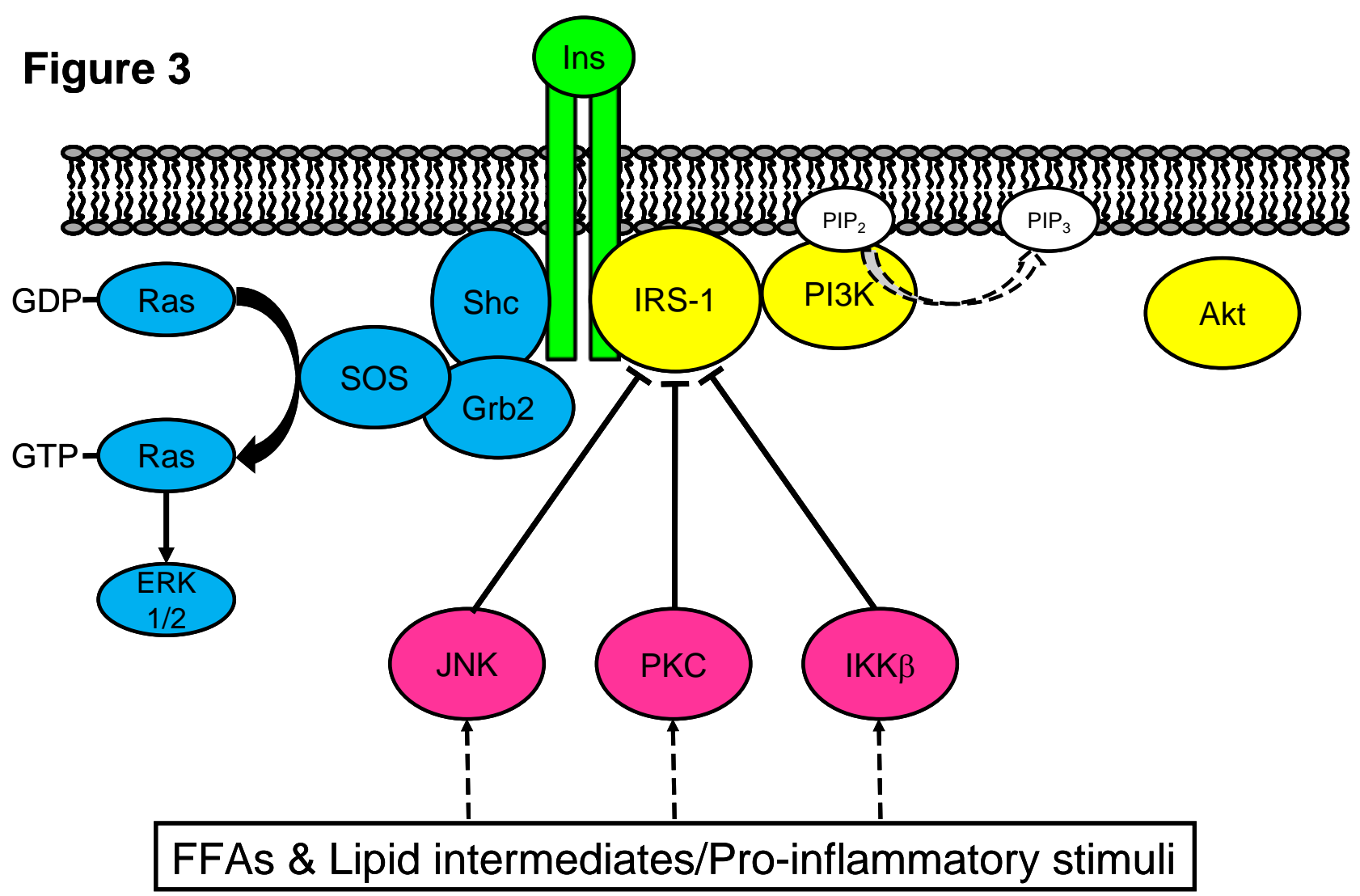

Figure 3: Molecular mechanism of selective insulin resistance

Insulin resistance is reported to be due to metabolites of ectopic lipid, increased free fatty acid (FFA) concentrations and proinflammatory cytokines stimulating one or more of the serine/threonine protein kinases protein kinase $\mathrm{C}(\mathrm{PKC}), \mathrm{C}-\mathrm{J}$ un $\mathrm{N}$-terminal kinase (J NK) and inhibitor of NF8B kinase ${ }^{2}\left(\right.$ IKK $\left.{ }^{2}\right)$. Activated PKC, JNK and IKK ${ }^{2}$ phosphorylate insulin receptor substrate-1 (IRS-1) at inhibitory residues, inhibiting activation by insulin. As a consequence, Akt activation is suppressed, whereas insulin stimulation of ERK1/2 is unaffected. Akt, protein kinase B; Grb2, growth factor receptor-bound protein 2; PI3K, phosphatidylinositol 3'-kinase; $\mathrm{PIP}_{2}$, phosphatidylinositol-4,5-bisphosphate; $\mathrm{PIP}_{3}$, phosphatidylinositol-3,4,5-trisphosphate; Shc, Src-homology and collagen-like; SOS, Son of Sevenless. 
